\title{
Dynamic Virtual Machines Placement in a Cloud Environment by Multi-Objective Programming
}

\author{
Hanying Kao*, Yumin Yang \\ Department of Computer Science and Information Engineering, National Dong Hwa University. No. 1, Sec. 2, \\ Da-Hsueh Rd., Shoufeng, Hualien 97401, Taiwan.
}

* Corresponding author. Tel.:+886-3-8634059; email: teresak@gms.ndhu.edu.tw

Manuscript submitted January 16, 2017; accepted June 24, 2017.

doi: 10.177606/ijcce.2017.6.3.193-200

\begin{abstract}
Recently, cloud services and cloud computing have revolutionized both academic research and industrial practices. A corresponding focus on how to improve the performance of cloud computing is growing apace. It is a significant approach to allocate virtual machines (VMs) on a set of physical machines (PMs). Computing resources can be utilized effectively with the optimal distribution of the virtual machines among the physical machines. This study aims to establish the dynamic placement model of VMs by multi-objective programming (MOP) for minimizing energy consumption, maximizing effectiveness of physical machine, and minimizing the task waiting time. The genetic algorithm (GA) is used to solve the multi-objective programming models and compared with the greedy method (GM). Experiments are implemented to verify the effectiveness of the proposed methods.
\end{abstract}

Key words: Virtual machine placement, cloud computing, multi-objective programming, genetic algorithm, greedy method.

\section{Introduction}

Recently, cloud services and cloud computing [1]-[3] have revolutionized both academic research and industrial practices. A corresponding focus on how to improve the performance of cloud computing is growing apace. It is a significant approach to allocate virtual machines (VMs) on a set of physical machines (PMs) [4]-[7]. Computing resources can be utilized effectively with the optimal distribution of the virtual machines among the physical machines. This study aims to establish the dynamic placement model of VMs by multi-objective programming (MOP) for minimizing energy consumption, maximizing effectiveness of physical machine, and minimizing the task waiting time. Experiments are implemented to verify the effectiveness of the proposed methods.

\section{Methods}

Consider a set of virtual machines to be allocated in another set of physical machines (servers) in successive periods. The parameters and variables are defined in Table 1.

\subsection{The multi-Objective Programming Model}

Based on the static placement model in cloud computing [4], the model intends to extend the model into a dynamic one and solve the dynamic allocation problem by multi-objective programming. The model is designed as below: 


$$
\begin{gathered}
\min Z_{1}=\sum_{t=1}^{l} \sum_{j=1}^{m} P_{j}=\sum_{t=1}^{l} \sum_{j=1}^{m}\left(y_{j t}\left(\left(P_{j}^{\max }-P_{j}^{m i n}\right) \sum_{i=1}^{n}\left(x_{i j t} R_{C P U_{i t}}\right)+P_{j}^{m i n}\right)\right) \\
\min Z_{2}=\sum_{t=1}^{l} \sum_{j=1}^{m} W_{j}=\sum_{t=1}^{l} \sum_{j=1}^{m}\left(y_{j t} \frac{\mid\left(T_{C P U_{j}}-\sum_{i=1}^{n}\left(x_{i j t} R_{C P U_{i t}}\right)\right)-\left(T_{m_{j}}-\sum_{i=1}^{n}\left(x_{i j t} R_{m_{i t}}\right) \mid\right.}{\sum_{i=1}^{n}\left(x_{i j t} R_{C P U_{i t}}\right)+\sum_{i=1}^{n}\left(x_{i j t} R_{m_{i t}}\right)}\right) \\
\min Z_{3}=\sum_{t=1}^{l} \operatorname{Time}_{t}=\alpha \cdot \max \left\{y_{j t} \cdot \sum_{i=1}^{n}\left(x_{i j t} R_{C P U_{i t}}\right)\right\}
\end{gathered}
$$

s.t.

$$
\begin{gathered}
\sum_{t=1}^{l} \sum_{j=1}^{m} x_{i j t}=1, \forall i \in I \\
\sum_{t=1}^{l} \sum_{i=1}^{n} x_{i j t} R_{C P U_{i t}} \leq y_{j t} T_{C P U_{j t}}, \forall j \in J \\
\sum_{t=1}^{l} \sum_{i=1}^{n} x_{i j t} R_{m_{j t}} \leq y_{j t} T_{m_{j t}}, \forall j \in J \\
x_{i j t}, y_{j t} \in\{0,1\} \quad \forall i \in I, \forall j \in J, \forall t \in T .
\end{gathered}
$$

Table 1. Parameters and Variables

\begin{tabular}{cc}
\hline \hline Notation & Description \\
\hline$I$ & The index set of virtual machines \\
$L$ & The index set of physical machines \\
$n$ & The index set of periods \\
$l$ & The number of virtual machines \\
The number of physical machines & The number of periods \\
$R_{C P U_{i t}}$ & The CPU demand of each virtual machine at period $t$ \\
$R_{m_{i t}}$ & The memory demand of each virtual machine at period $t$ \\
$T_{C P U_{j}}$ & The threshold of the CPU utilization with each server \\
$T_{m_{j}}$ & The threshold of the memory utilization with each server \\
$P_{j}^{m a x}$ & The maximal power value the $j$-th server can be utilized \\
$P_{j}^{m i n}$ & The minimal power value when the $j$-th server is utilized \\
$\alpha$ & The conversion constant for waiting time and CPU utilization \\
$x_{i j t}$ & The binary variable indicating if VM $i$ is assigned to PM $j$ at period $t$ \\
$y_{j t}$ & The binary variable indicating if PM $j$ is in use at period $t$ \\
\hline \hline
\end{tabular}

The model minimizes the total power consumption in (1), minimizes the power wastage in (2), and minimizes the waiting time of the processes in (3). Constraint (4) assigns every virtual machine to only one physical server in every period. Inequalities (5) and (6) ensure that the usages of CPU and memory are under their capacities in the execution duration. Constraints (7) and (8) define $x_{i j t}, y_{j t}$ as binary variables representing the association of VM $i$ and server $j$ in time $t$, and the activation of server $j$, respectively.

\subsection{The Solution Processes}

Many techniques have been proposed for solving multi-objective programming problems, and some of 
them are inspired by Zimmermann's fuzzy approach [8]. This study develops the solution process based on Zimmermann's approach to solve the model as (1) - (7).

Step 1. Get the ideal solution of each objective.

To obtain the ideal solution $z_{i}^{*}$, each objective function is minimized independently regardless of other objectives.

Step 2. Get the anti-ideal solution of each objective.

To obtain the anti-ideal solution $z_{i}^{-}$, each objective function is maximized independently regardless of other objectives.

Step 3. Define the membership function of each objective by its ideal and anti-ideal solutions as below.

$$
\mu_{z_{i}}\left(z_{i}\right)=\frac{z_{i}-z_{i}^{-}}{z_{i}^{*}-z_{i}^{-}}
$$

The membership function evaluates the fulfillment level of each objective.

Step 4. Get the final solution.

The final solution is found by maximizing the total satisfaction level as below.

$$
\begin{aligned}
& \lambda=\mu_{z_{1}}+\mu_{z_{2}}+\mu_{z_{3}} \\
& \text { s.t. }(4)-(7)
\end{aligned}
$$

The flowchart of the solution processes is illustrated as in Fig. 1.

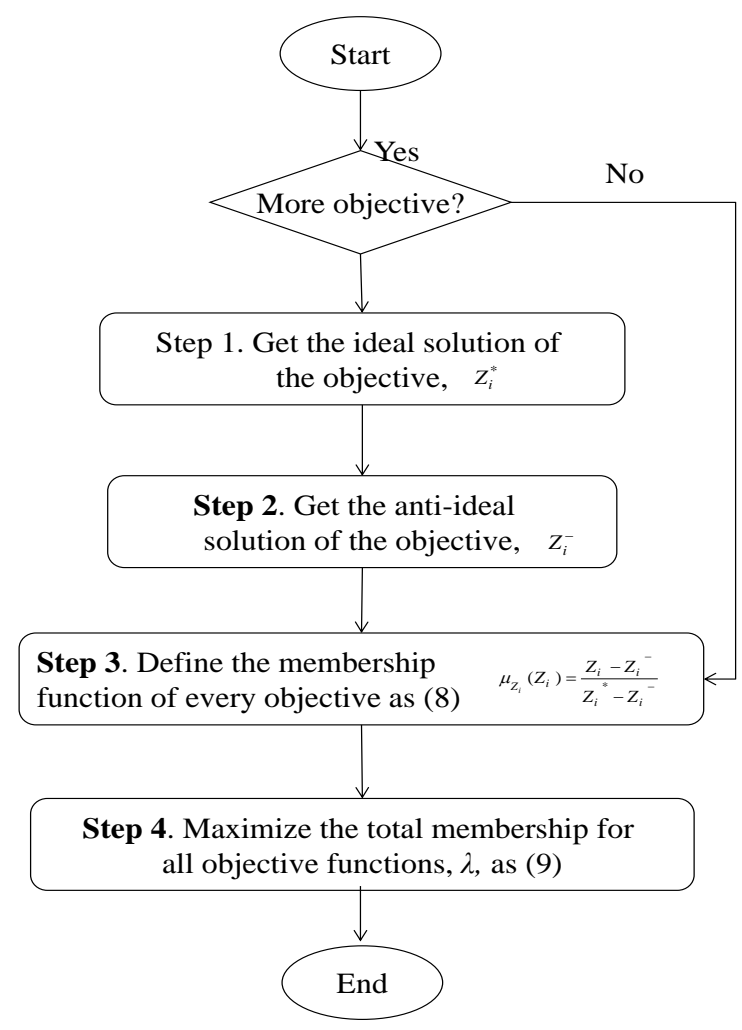

Fig. 1. The solution processes of MOP.

This study uses the genetic algorithm [9], [10] to carry out the computation for MOP. Genetic algorithm 
(GA) is a meta-heuristic method inspired by genetic evolution for combinational optimization as described below. It is a practical approach in common use to solve complex computational problems in information and communication technology.

\section{Algorithm-GA}

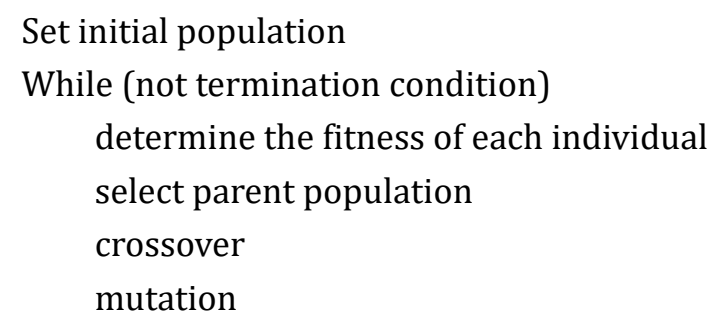

End while

On the other hand, the greedy method [11], [12] is implemented for comparison.

\section{Experiments and Results}

Consider a set of virtual machines to be allocated in another set of physical machines (servers) in two successive periods. This study assumes identical specifications for all physical servers equipped with Pentium(R) Dual-Core E5300 / $2.6 \mathrm{GHz}$ processor, 2 GB memory. Assume that every physical server can satisfy the requirements of any virtual machines. This work randomly generates 25 virtual machine samples and expresses their requirements as the utilization rate of CPU and memory in Table 2. Three scenarios of experiments are conducted using randomly selected subsets of the samples in Case 1: 15 VMs to 10 PMs; Case 2: 20 VMs to 10 PMs; and Case 3: 25 VMs to 7 PMs. The solutions and detailed results of the three cases are summarized in Table 3-5, and Table 6-8, respectively.

In Case 1 (Table 3), the multi-objective programming with genetic algorithm (MOP-GA) outperforms the greedy method (GM) in power wastage $\left(Z_{2}\right)$ and waiting time $\left(Z_{3}\right)$. In case 2 (Table 4), the MOP-GA obtains lower power consumption $\left(Z_{1}\right)$ and power wastage $\left(Z_{2}\right)$, but generate longer waiting time $\left(Z_{3}\right)$. In case 3 (Table 5), the GM outperforms the MOP-GA except in power wastage $\left(Z_{2}\right)$. However, in case 3 the computing requirements of the virtual machines exceed the resources of the physical servers, so several virtual machines are unallocated (as shown in Table 8). In Case 3, 6 virtual machines $(11,15,19,20,21,24)$ are unallocated with the GM, and 3 virtual machines $(10,16,21)$ are unallocated with the MOP-GA. That is, the MOP-GA generates higher completion compared to the greedy method.

Table 2. Virtual Machine Samples

\begin{tabular}{lll|lll}
\hline \hline VM & CPU usage & $\begin{array}{c}\text { Memory } \\
\text { Usage }\end{array}$ & VM & CPU usage & $\begin{array}{c}\text { Memory } \\
\text { Usage }\end{array}$ \\
\hline 1 & 0.48 & 0.71 & 2 & 0.01 & 0.44 \\
3 & 0.64 & 0.46 & 4 & 0.02 & 0.41 \\
5 & 0.45 & 0.61 & 6 & 0.39 & 0.03 \\
7 & 0.81 & 0.46 & 8 & 0.46 & 0.47 \\
9 & 0.15 & 0.37 & 10 & 0.73 & 0.56 \\
11 & 0.83 & 0.46 & 12 & 0.21 & 0.24 \\
13 & 0.33 & 0.42 & 14 & 0.28 & 0.31 \\
15 & 0.84 & 0.79 & 16 & 0.62 & 0.5 \\
17 & 0.59 & 0.51 & 18 & 0.33 & 0.26 \\
19 & 0.45 & 0.89 & 20 & 0.56 & 0.87 \\
21 & 0.67 & 0.82 & 22 & 0.52 & 0.47 \\
23 & 0.01 & 0.4 & 24 & 0.81 & 0.89 \\
25 & 0.46 & 0.53 & & & \\
\hline \hline
\end{tabular}


Table 3. Solutions to Case 1 (15 VMs to $10 \mathrm{PMs}$ )

\begin{tabular}{ll}
\hline \hline By genetic algorithm & By greedy method \\
\hline $\begin{array}{l}Z_{1}=2133.39, Z_{2}=0.8971, Z_{3}=1.1 \\
\text { For } t=1\end{array}$ & $Z_{1}=2133.39, Z_{2}=1.9539, Z_{3}=1.26$ \\
$\quad y_{1,1}=1, y_{2,1}=1, y_{3,1}=1, y_{4,1}=1, y_{5,1}=1, y_{6,1}=1, y_{7,1}=1, y_{8,1}=0$, & $y_{1,1}=1, y_{2,1}=1, y_{3,1}=1, y_{4,1}=1, y_{5,1}=1, y_{6,1}=1, y_{7,1}=1$, \\
$y_{9,1}=1, y_{10,1}=1, x_{1,5,1}=1, x_{3,2,1}=1, x_{5,6,1}=1, x_{6,3,1}=1, x_{8,10,1}=1$, & $y_{8,1}=1, y_{9,1}=1, y_{10,1}=1, x_{1,7,1}=1, x_{2,1,1}=1, x_{3,6,1}=1$, \\
$x_{9,3,1}=1, x_{10,1,1}=1, x_{12,4,1}=1, x_{13,3,1}=1, x_{14,7,1}=1, x_{15,9,1}=1$, all & $x_{4,1,1}=1, x_{5,5,1}=1, x_{6,1,1}=1, x_{7,8,1}=1, x_{8,4,1}=1, x_{9,2,1}=1$, \\
remaining $x_{i j 1}=0$ & $\begin{array}{l}x_{10,9,1}=1, x_{11,10,1}=1, x_{12,2,1}=1, x_{13,3,1}=1, x_{14,3,1}=1, \text { all } \\
\text { remaining } x_{i j 1}=0\end{array}$ \\
& \\
For $t=2$ & \\
$y_{1,2}=0, y_{2,2}=0, y_{3,2}=0, y_{4,2}=1, y_{5,2}=0, y_{6,2}=0, y_{7,2}=1, y_{8,2}=0$, & $y_{1,2}=1, y_{2,2}=0, y_{3,2}=0, y_{4,2}=0, y_{5,2}=0, y_{6,2}=0, y_{7,2}=0$, \\
$y_{9,2}=0, y_{10,2}=0 x_{2,7,2}=1, x_{4,4,2}=1, x_{7,7,2}=1, x_{11,4,2}=1$, all remaining & $y_{8,2}=0, y_{9,2}=0, y_{10,2}=0$ \\
$x_{i j 2}=0$ & $x_{15,1,2}=1$, all remaining $x_{i j 2}=0$ \\
\hline
\end{tabular}

Table 4. Solutions to Case 2 (20 VMs to $10 \mathrm{PMs}$ )

\begin{tabular}{ll}
\hline \hline By genetic algorithm & By greedy method \\
\hline $\begin{array}{l}Z_{1}=2592.54, Z_{2}=1.3739, Z_{3}=1.61 \\
\text { For } t=1\end{array}$ & $Z_{1}=2916.54, Z_{2}=2.6749, Z_{3}=1.35$ \\
$\quad y_{1,1}=0, y_{2,1}=1, y_{3,1}=1, y_{4,1}=1, y_{5,1}=1, y_{6,1}=1$, & $y_{1,1}=1, y_{2,1}=1, y_{3,1}=1, y_{4,1}=1, y_{5,1}=1, y_{6,1}=1, y_{7,1}=1, y_{8,1}=1$, \\
$y_{7,1}=1, y_{8,1}=1, y_{9,1}=1, y_{10,1}=0, x_{1,6,1}=1, x_{2,7,1}=1$, & $y_{9,1}=1, y_{10,1}=1, x_{1,9,1}=1, x_{2,1,1}=1, x_{3,6,1}=1, x_{4,1,1}=1, x_{5,5,1}=1$, \\
$x_{4,9,1}=1, x_{5,5,1}=1, x_{7,7,1}=1, x_{8,4,1}=1, x_{11,9,1}=1$, & $x_{6,1,1}=1, x_{7,10,1}=1, x_{8,4,1}=1, x_{9,2,1}=1, x_{12,2,1}=1, x_{13,4,1}=1$, \\
$x_{12,3,1}=1, x_{15,8,1}=1, x_{17,3,1}=1, x_{18,5,1}=1, x_{19,2,1}=1$, all & $x_{14,3,1}=1, x_{16,7,1}=1, x_{17,8,1}=1, x_{18,3,1}=1$, all remaining $x_{i j 1}=0$ \\
remaining $x_{i j 1}=0$ & \\
& \\
For $t=2$ & \\
$y_{1,2}=0, y_{2,2}=0, y_{3,2}=1, y_{4,2}=1, y_{5,2}=1, y_{6,2}=1, y_{7,2}=1$, & $y_{1,2}=1, y_{2,2}=1, y_{3,2}=1, y_{4,2}=1, y_{5,2}=1, y_{6,2}=0, y_{7,2}=0, y_{8,2}=0$, \\
$y_{8,2}=0, y_{9,2}=0, y_{10,2}=0$, & $y_{9,2}=0, y_{10,2}=0$ \\
$x_{3,7,2}=1, x_{6,3,2}=1, x_{9,7,2}=1, x_{10,5,2}=1, x_{13,3,2}=1$, & $x_{10,1,2}=1, x_{11,2,2}=1, x_{15,5,2}=1, x_{19,3,2}=1, x_{20,4,2}=1$, all \\
$x_{14,6,2}=1, x_{16,6,2}=1, x_{20,4,2}=1$, & remaining $x_{i j 2}=0$ \\
all remaining $x_{i j 2}=0$ & \\
\hline \hline
\end{tabular}

Table 5. Solutions to Case 3 (25 VMs to 7 PMs)

\begin{tabular}{|c|c|}
\hline By genetic algorithm & By greedy method \\
\hline $\begin{array}{l}Z_{1}=2778.39, Z_{2}=1.2545, Z_{3}=1.65 \\
\text { For } t=1\end{array}$ & $Z_{1}=2664.97, Z_{2}=2.3243, Z_{3}=1.33$ \\
\hline $\begin{array}{l}y_{1,1}=1, y_{2,1}=1, y_{3,1}=1, y_{4,1}=1, y_{5,1}=1, y_{6,1}=1, y_{7,1}=1, \\
\quad x_{1,7,1}=1, x_{2,3,1}=1, x_{7,2,1}=1, x_{8,6,1}=1, x_{9,1,1}=1, \\
x_{11,3,1}=1, \\
\quad x_{14,4,1}=1, x_{17,1,1}=1, x_{19,5,1}=1, x_{23,2,1}=1, x_{25,4,1}=1, \\
\text { all remaining } x_{i j 1}=0\end{array}$ & $\begin{array}{l}y_{1,1}=1, y_{2,1}=1, y_{3,1}=1, y_{4,1}=1, y_{5,1}=1, y_{6,1}=1, y_{7,1}=1, \\
x_{2,2,1}=1, x_{4,1,1}=1, x_{5,8,1}=1, x_{6,1,1}=1, x_{7,10,1}=1, x_{8,5,1}=1, \\
x_{9,3,1}=1, x_{12,2,1}=1, x_{13,4,1}=1, x_{14,3,1}=1, x_{17,6,1}=1, x_{18,4,1}=1, \\
x_{23,1,1}=1, x_{25,7,1}=1 \text { all remaining } x_{i j 1}=0\end{array}$ \\
\hline For $t=2$ & \\
\hline $\begin{array}{c}y_{1,2}=1, y_{2,2}=1, y_{3,2}=1, y_{4,2}=1, y_{5,2}=1, y_{6,2}=1, y_{7,2}=1, \\
x_{3,1,2}=1, x_{4,2,2}=1, x_{5,6,2}=1, x_{6,2,2}=1, x_{12,1,2}=1\end{array}$ & $y_{1,2}=1, y_{2,2}=1, y_{3,2}=1, y_{4,2}=1, y_{5,2}=1, y_{6,2}=1, y_{7,2}=1$, \\
\hline $\begin{array}{l}x_{13,5,2}=1, x_{15,7,2}=1, x_{18,6,2}=1, \\
x_{20,4,2}=1, x_{22,5,2}=1, x_{24,3,2}=1, \text { all remaining } x_{i j 2}=0\end{array}$ & $\begin{array}{l}x_{1,5,2}=1, x_{3,2,2}=1, x_{5,1,2}=1, x_{7,6,2}=1, x_{10,7,2}=1, x_{16,3,2}=1, x_{17,4,2}=1 \\
\text { all remaining } x_{i j 2}=0\end{array}$ \\
\hline
\end{tabular}

\section{Concluding Remarks}

This study establishes the dynamic placement model of VMs by multi-objective programming for 
minimizing energy consumption, maximizing effectiveness of physical machine, and minimizing the task waiting time. The multi-objective programming with genetic algorithm and the greedy method are compared in solving the placement models. Three scenarios are prepared to test the feasibility and effectiveness of the model. Based on the results, the proposed MOP model is effective the virtual machine placement in cloud computing environments considering both power efficiency and allocation performance. In summary, the multi-objective programming with genetic algorithm outperforms the greedy method when the physical servers provide sufficient resources (case 1 and case 2) and higher throughput under insufficient computing resources (case 3). Without loss of generality, the two-period setting can be extended to multi-period scenarios. For future studies, various objective functions and algorithms can be consolidated to raise the precision and robustness of the models.

Table 6. Results of Case 1 (15 VMs to $10 \mathrm{PMs}$ )

\begin{tabular}{|c|c|c|c|c|c|c|}
\hline & VM allocated & CPU Usage & Memory Usage & VM allocated & CPU Usage & Memory Usage \\
\hline & \multicolumn{3}{|c|}{ By MOP-GA } & \multicolumn{3}{|c|}{ By GM } \\
\hline$t=1$ & & & & & & \\
\hline PM1 & 10 & 0.73 & 0.56 & $2,4,6$ & 0.42 & 0.88 \\
\hline PM2 & 3 & 0.64 & 0.46 & 9,12 & 0.36 & 0.61 \\
\hline PM3 & $6,9,13$ & 0.87 & 0.82 & 13,14 & 0.61 & 0.73 \\
\hline PM4 & 12 & 0.21 & 0.24 & 8 & 0.46 & 0.47 \\
\hline PM5 & 1 & 0.48 & 0.71 & 5 & 0.45 & 0.61 \\
\hline PM6 & 5 & 0.45 & 0.61 & 3 & 0.64 & 0.46 \\
\hline PM7 & 14 & 0.28 & 0.31 & 1 & 0.48 & 0.71 \\
\hline PM8 & - & 0 & 0 & 7 & 0.81 & 0.46 \\
\hline PM9 & 15 & 0.84 & 0.79 & 10 & 0.73 & 0.56 \\
\hline PM10 & 8 & 0.46 & 0.47 & 11 & 0.83 & 0.46 \\
\hline \multicolumn{7}{|l|}{$t=2$} \\
\hline PM1 & - & 0 & 0 & 15 & 0.84 & 0.79 \\
\hline PM2 & - & 0 & 0 & - & 0 & 0 \\
\hline PM3 & - & 0 & 0 & - & 0 & 0 \\
\hline PM4 & 4,11 & 0.85 & 0.87 & - & 0 & 0 \\
\hline PM5 & - & 0 & 0 & - & 0 & 0 \\
\hline PM6 & - & 0 & 0 & - & 0 & 0 \\
\hline PM7 & 2,7 & 0.82 & 0.9 & - & 0 & 0 \\
\hline PM8 & - & 0 & 0 & - & 0 & 0 \\
\hline PM9 & - & 0 & 0 & - & 0 & 0 \\
\hline PM10 & - & 0 & 0 & - & 0 & 0 \\
\hline
\end{tabular}

Table 7. Results of Case 2 (20 VMs to $10 \mathrm{PMs}$ )

\begin{tabular}{|c|c|c|c|c|c|c|}
\hline & VM allocated & CPU Usage & Memory Usage & VM allocated & CPU Usage & Memory Usage \\
\hline & \multicolumn{3}{|c|}{ By MOP-GA } & \multicolumn{3}{|c|}{ By GM } \\
\hline$t=1$ & & & & & & \\
\hline PM1 & - & 0 & 0 & $2,4,6$ & 0.42 & 0.88 \\
\hline PM2 & 19 & 0.45 & 0.89 & 9,12 & 0.36 & 0.61 \\
\hline PM3 & 12,17 & 0.8 & 0.75 & 14,18 & 0.61 & 0.57 \\
\hline PM4 & 8 & 0.46 & 0.47 & 8,13 & 0.79 & 0.89 \\
\hline PM5 & 5,18 & 0.78 & 0.87 & 5 & 0.45 & 0.61 \\
\hline PM6 & 1 & 0.48 & 0.71 & 3 & 0.64 & 0.46 \\
\hline PM7 & 2,7 & 0.82 & 0.9 & 16 & 0.62 & 0.5 \\
\hline PM8 & 15 & 0.84 & 0.79 & 17 & 0.59 & 0.51 \\
\hline PM9 & 4,11 & 0.85 & 0.87 & 1 & 0.48 & 0.71 \\
\hline PM10 & - & 0 & 0 & 7 & 0.81 & 0.46 \\
\hline$t=2$ & & & & & & \\
\hline PM1 & - & 0 & 0 & 10 & 0.73 & 0.56 \\
\hline PM2 & - & 0 & 0 & 11 & 0.83 & 0.46 \\
\hline PM3 & 6,13 & 0.72 & 0.45 & 19 & 0.45 & 0.89 \\
\hline PM4 & 20 & 0.56 & 0.87 & 20 & 0.56 & 0.87 \\
\hline PM5 & 10 & 0.73 & 0.56 & 15 & 0.84 & 0.79 \\
\hline PM6 & 14,16 & 0.9 & 0.81 & - & 0 & 0 \\
\hline PM7 & 3,9 & 0.79 & 0.83 & - & 0 & 0 \\
\hline PM8 & - & 0 & 0 & - & 0 & 0 \\
\hline PM9 & - & 0 & 0 & - & 0 & 0 \\
\hline PM10 & - & 0 & 0 & - & 0 & 0 \\
\hline
\end{tabular}


Table 8. Results of Case 3 (25 VMs to 7 PMs)

\begin{tabular}{|c|c|c|c|c|c|c|}
\hline & VM allocated & CPU Usage & Memory Usage & VM allocated & CPU Usage & Memory Usage \\
\hline & \multicolumn{3}{|c|}{ By MOP-GA } & \multicolumn{3}{|c|}{ By GM } \\
\hline \multicolumn{7}{|l|}{$t=1$} \\
\hline PM1 & 9,17 & 0.74 & 0.88 & $4,6,23$ & 0.42 & 0.88 \\
\hline PM2 & 7,23 & 0.82 & 0.86 & 2,12 & 0.36 & 0.61 \\
\hline PM3 & 2,11 & 0.84 & 0.9 & 9,14 & 0.61 & 0.57 \\
\hline PM4 & 14,25 & 0.74 & 0.84 & 13,18 & 0.79 & 0.89 \\
\hline PM5 & 19 & 0.45 & 0.89 & 8 & 0.45 & 0.61 \\
\hline PM6 & 8 & 0.46 & 0.47 & 17 & 0.64 & 0.46 \\
\hline PM7 & 1 & 0.48 & 0.71 & 25 & 0.62 & 0.5 \\
\hline \multicolumn{7}{|l|}{$t=2$} \\
\hline PM1 & 3,12 & 0.85 & 0.7 & 5 & 0.59 & 0.51 \\
\hline PM2 & 4,6 & 0.41 & 0.44 & 3 & 0.48 & 0.71 \\
\hline PM3 & 24 & 0.81 & 0.89 & 16 & 0.81 & 0.46 \\
\hline PM4 & 20 & 0.56 & 0.87 & 17 & 0.73 & 0.56 \\
\hline PM5 & 13,22 & 0.85 & 0.89 & 1 & 0.83 & 0.46 \\
\hline PM6 & 5,18 & 0.78 & 0.87 & 7 & 0.45 & 0.89 \\
\hline PM7 & 15 & 0.84 & 0.79 & 10 & 0.56 & 0.87 \\
\hline Unallocated & \multicolumn{3}{|c|}{$10,16,21$} & \multicolumn{3}{|c|}{$11,15,19,20,21,24$} \\
\hline
\end{tabular}

\section{Acknowledgment}

The authors appreciate the anonymous referees for their helpful comments and suggestions to improve the quality of this manuscript. Also we are indebted to the Ministry of Science and Technology, Taiwan for financially supporting this research under grant No. 103-2410-H-259-084- (H.-Y. Kao). The brief portion of this work has been presented in International Conference on Intelligent Informatics and BioMedical Sciences 2015 (iciibms 2015), Okinawa, Japan.

\section{References}

[1] Abadi, D. J. (2009). Data management in the cloud: Limitations and opportunities. IEEE Data Engineering Bulletin, 32, 3-12.

[2] Chen, C. L. P., \& Zhang, C. Y. (2014). Data-intensive applications, challenges, techniques and technologies: A survey on big data. Information Sciences, 275(1), 314-347.

[3] Guazzelli, A., Stathatos, K., \& Zeller, M. (2009). Efficient deployment of predictive analytics through open standards and cloud computing. ACM SIGKDD Explorations Newsletter, 11(1), 32-38.

[4] Gao, Y., Guan, H., Qi, Z., Hou, Y., \& Liu, L. (2013). A multi-objective ant colony system algorithm for virtual machine placement in cloud computing. Journal of Computer and System Sciences, 79(8), 1230-1242.

[5] Ramezani, F., Lu, J., \& Hussain, F. K. (2014). Task-based system load balancing in cloud computing using particle swarm optimization. International Journal of Parallel Programming, 42(5), 739-754.

[6] Zhao, Y., Huang, Y., Chen, K., Yu, Wang, M., S., \& Li, D. S. (2015). Joint VM placement and topology optimization for traffic scalability in dynamic datacenter networks. Computer Networks, 80, 109-123.

[7] Coutinho, R. C., Drummond, L. M. A., Frota, Y., \& Oliveira, D. (2015). Optimizing virtual machine allocation for parallel scientific workflows in federated clouds. Future Generation Computer Systems, 46, 51-68.

[8] Zimmermann, H. J. (1978). Fuzzy programming and linear programming with several objective functions. Fuzzy Sets and Systems, 1(1), 45-55.

[9] Kaaouache, M. A., \& Bouamama, S. (2015). Solving bin packing problem with a hybrid genetic algorithm for VM placement in cloud. Procedia Computer Science, 60, 1061-1069.

[10] Masdari, M., Nabavi, S. S., \& Ahmadi, V. (2016). An overview of virtual machine placement schemes in 
cloud computing. Journal of Network and Computer Applications, 66, 106-127.

[11] Omara, F. A., Khattab, S. M., \& Sahal, R. (2014). Optimum resource allocation of database in cloud computing. Egyptian Informatics Journal, 15(1), 1-12.

[12] Palmieri, F., Fiore, U., Ricciardi, S., \& Castiglione, A. (2016). GRASP-based resource re-optimization for effective big data access in federated clouds. Future Generation Computer Systems, 54, 168-179.

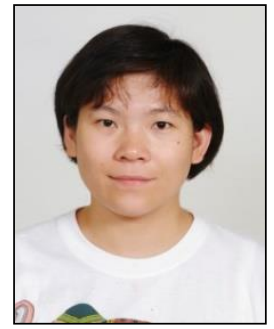

Hanying Kao is currently the professor with the Department of Computer Science and Information Engineering, National Dong Hwa University, Hualien, Taiwan. She received her Ph.D. degree in Information Management from National Chiao Tung University, Taiwan. Dr. Kao has rich experiences in manufacturing information systems and related academic research. Her current interests include multi-objective decision methods, knowledge modeling, fuzzy optimization, etc. Her works have been published with numerous international journal and conferences.

Yumin Yang received his master degree in computer science and information engineering, National Dong Hwa University, Hualien, Taiwan. His current interests include multi-objective decision methods, cloud computing, knowledge based systems, etc. 